\title{
Calcium Phosphate Cement "Space Fill-in" Augmentation in Autologous Cranioplasty for Large Cranial Defect: Additional Technical Consideration and Its Long-term Follow-up
}

\author{
Ichiro TAKUMI ${ }^{1}$ and Masataka AKIMOTO² \\ ${ }^{1}$ Department of Neurosurgery, St. Marianna University School of Medicine, Kawasaki, \\ Kanagawa, Japan \\ ${ }^{2}$ Department of Plastic Surgery, Nippon Medical School Chiba Hokuso Hospital, Inzai, \\ Chiba, Japan
}

\begin{abstract}
In the skull tumor surgery that requires a large cranial reconstruction, economical one-time surgery is challenging. Calcium phosphate paste (CPC) alone is not applied in the large defect. Other plastic fill-in materials have each drawback. Ready-made implants are costly. The authors present additional technique of CPC cranioplasty combined with mainstay autologous grafts for a large cranial defect. The combination of split rib grafts was augmented by CPC. Tenons were placed for the stability of grafts. Our newly additional technique is that CPC is filled in the small adjacent spaces of autografts, not applied as the simple on-lay graft. We introduced this method to a 57-year-old gentleman with left parietal expansile skull tumor. The aesthetics of the patient has been satisfactory, and there were no complaints about pain in the graft site. In the follow-up period of 8 years, both autologous grafts and CPC were well maintained without marked resorption. This patient could work as a farmer in this period. Our methods fulfilled the requirements of aesthetics and in-situ plasticity for a larger cranial defect.
\end{abstract}

Keywords: autologous, calcium phosphate cement, aesthetic, rib grafts, calvarial grafts

\section{Introduction}

Several ready-made alloplastic implants such as hydroxy apatite (HA), polyether ether ketone (PEEK), titanium and polyethylene are frequently used for cranial reconstruction. Poly methyl-meta acrylate (PMMA) paste used to be used as it has good strength, but it is not used these days in Japan with the frequent drawbacks of post-surgical infection and skin trouble. ${ }^{1)}$ PEEK may have higher local complication rate, ${ }^{1)}$ but so as in thinner titanium mesh implants, ${ }^{2)}$ or fracture is observed in HA implants. ${ }^{3)}$ Patient-specific titanium-reinforced calcium phosphate implant ${ }^{4)}$ solves some of these problems; however, it is costly to make those implants. Overall, accessibility to the various materials may differ in

Received October 18, 2020; Accepted December 4, 2020

Copyright $@ 2021$ by The Japan Neurosurgical Society This work is licensed under a Creative Commons AttributionNonCommercial-NoDerivatives International License. countries to countries, for instance, PEEK has never been available in Japan to date.

Paste fill-in materials have been used in skull reconstruction because of its in-situ plasticity. Calcium phosphate paste (CPC) is frequently used in the augmentation of cranial reconstruction. ${ }^{5,6)}$ CPC is not used near paranasal sinuses or after radiotherapy, to avoid further complications. CPC is not used in the large cranial defect cranioplasty alone. Its use should not exceed $25 \mathrm{~cm}^{2}$ of coverage area. ${ }^{5)}$ Autologous grafts ${ }^{7-9)}$ are, in a sense, available elsewhere, and eventually, economical.

We assumed that CPC cranioplasty designed for a large cranial defect may well be augmented with autologous graft of the Catcher's mask cranioplasty. ${ }^{8,9)}$ Catcher's mask cranioplasty is a "non-fully covered"
Part of this work was presented at the symposium of the 12th annual meeting of the Japan Society of Aesthetic Neurosurgery, on April13, 2019, Okayama Japan. 
cranioplasty which resembles a baseball catcher's mask, a combination cranioplasty of autologous grafts of rib grafts and/or calvarial grafts. These autografts function as mainstay to enable unfurling the sails of CPC grafts, to minimize the coverage area. Here, we report our technique of CPC cranioplasty augmentation of the Catcher's mask method, with a long-term follow-up.

\section{Technical Note}

This 57-year-old man noticed to the left parietal osseous mass at the time of traffic accident in 2006. As the patient complained an enlargement of the tumor in 2007, surgery was indicated because malignancy was suspected. CT scan shows $50 \mathrm{~mm} \times 49 \mathrm{~mm}$ lowdensity mass in the parietal bone, extending in the inner table to the outer table of calvaria (Figs. 1A-1D). N-butyl-2-cyanoacrylate (NBCA) was embolized into the tumor through the left superficial temporal artery three days prior to the surgical resection.

$8 \mathrm{~cm} \times 8 \mathrm{~cm}$ of craniotomy was placed in the left fronto-parietal lesion (Fig. 2A). As the dura matter was incised because of the invasion, it was substituted by free fascia lata graft (Fig. 2A). Split rib graft was harvested, arranged into parallel crosses by the 2-0 surgical nylon (Fig. 2B). Harvesting of split rib grafts is described elsewhere, ${ }^{9)}$ in brief, $10 \mathrm{~cm}$ of left 7 th and 9th ribs were selected not to take ones next to each other, harvested, and split by a reciprocating saw. The graft contour is created with utilizing the curve of rib. Eventually, one convex is compact bone and another convexity is aerated bone, and they are used to apply the double-crossed autologous graft of split rib grafts. Tenons were placed on the recipient skull (Fig. 2A, inset), to create the smooth contour by eliminating gaps, together with the stability of the graft application. These grafts were fixed with the 2-0 surgical nylon. $10 \mathrm{~mL}$ of CPC paste (NTK medical, Komaki, Aichi Japan) was prepared, thinned to form a plate shape by sandwiched in medical grade transparent dressings (Fig. 2C). One side of dressing was removed and CPC graft was placed onto the double-crossed autologous grafts (Figs. 2D and $2 \mathrm{E}$ ). The pathology revealed to be an intraosseous cavernous angioma of the parietal bone.

The grafts were assessed for 8 years after the surgery. There was no significant absorption in the autologous grafts (Figs. 1E, 1F, 1I, 1J, 1M, 1N, 1Q, 1R, $1 \mathrm{U}$, and $1 \mathrm{~V}$ ) or in the CPC (Figs. $1 \mathrm{G}, 1 \mathrm{~K}, 1 \mathrm{O}, 1 \mathrm{~S}$, and $1 \mathrm{~W}$ ). The aesthetical result is satisfactory (Fig. 3). Although there is no method to measure the strength of the graft, the graft fulfilled the needs to maintain his daily life that this gentleman continued his job as a farmer in the following 8 years after surgery. He complained no pain at the graft site (chest).

\section{Discussion}

In skull tumor surgery, one time cranioplasty with individually designed-skull implants is realized by neuro-navigator, ${ }^{10,11)}$ but some additional methods in-situ cranioplasty are introduced. Cranial defect of $4 \mathrm{~cm}$ diameter is reconstructed by CPC with a combination use of artificial implants with biological affinities, which works well in that size. ${ }^{12)}$ Titanium cranioplasty with in-situ CPC augmentation is reported, which may have some possibility of post-surgical infection. ${ }^{13)}$ A desktop 3D printer is used for the molding PMMA cost effectively, ${ }^{11,14)}$ and this might be a good solution if the in vitro molding lowers the previous disadvantages of PMMA cranioplasty technique. ${ }^{1}$ Our case was an expansile skull tumor. The tumor was removed with $1 \mathrm{~cm}$ margin and the attached dura matter was removed because we considered the possibility of its malignancy.

Catcher's mask ${ }^{8,9)}$ cranioplasty is characterized by the non-full-filling cranioplasty, and the advantage of this method is enabling larger defects-cranioplasty with limited amount of autologous grafts, with the advantage of cost-effectiveness. In the presented case, we used combined rib grafts for mainstay part, but combined rib-calvarium or calvarial grafts may be the other options to be considered. Post-surgical wound pain of the harvested site or the chest deformity should be considered and patient's informed consent should be acquired, but these managements are seldom required with the proper surgical technique. Intercostal neuralgia is avoided by designing the single skin incision along with the rib, not to across the intercostal nerve. Chest deformity is avoided by harvesting ribs every other one, like harvesting 5th rib and 7 th rib. There still maybe issues of aesthetics in autologous bone graft cranioplasty, but the technique is well established. ${ }^{15)} \mathrm{We}$ are limiting its application to the troubleshooting cranioplasty, such as repeated cranioplasty with 5-7 previous surgical intervention due to repeated failure of cranioplasty by implants infection or scalp erosion, as the strength of the autologous implants may not be sufficient as regular cranioplasty such as by preserved auto-bone or by ready-made cranial implants. Catcher's mask "framework" cranioplasty prevents patients from sinking skin flap syndrome, but strength wisely, even when the rim is well preserved, some part of patient's head is soft as there is no undercovering. We certainly consider other simple techniques of alloplastic cranial reconstruction before indicating our method, especially in the adult patients whose defect is pre-surgically measured.

Our method of rib harvesting is to take completely free graft without periosteum. There is an 


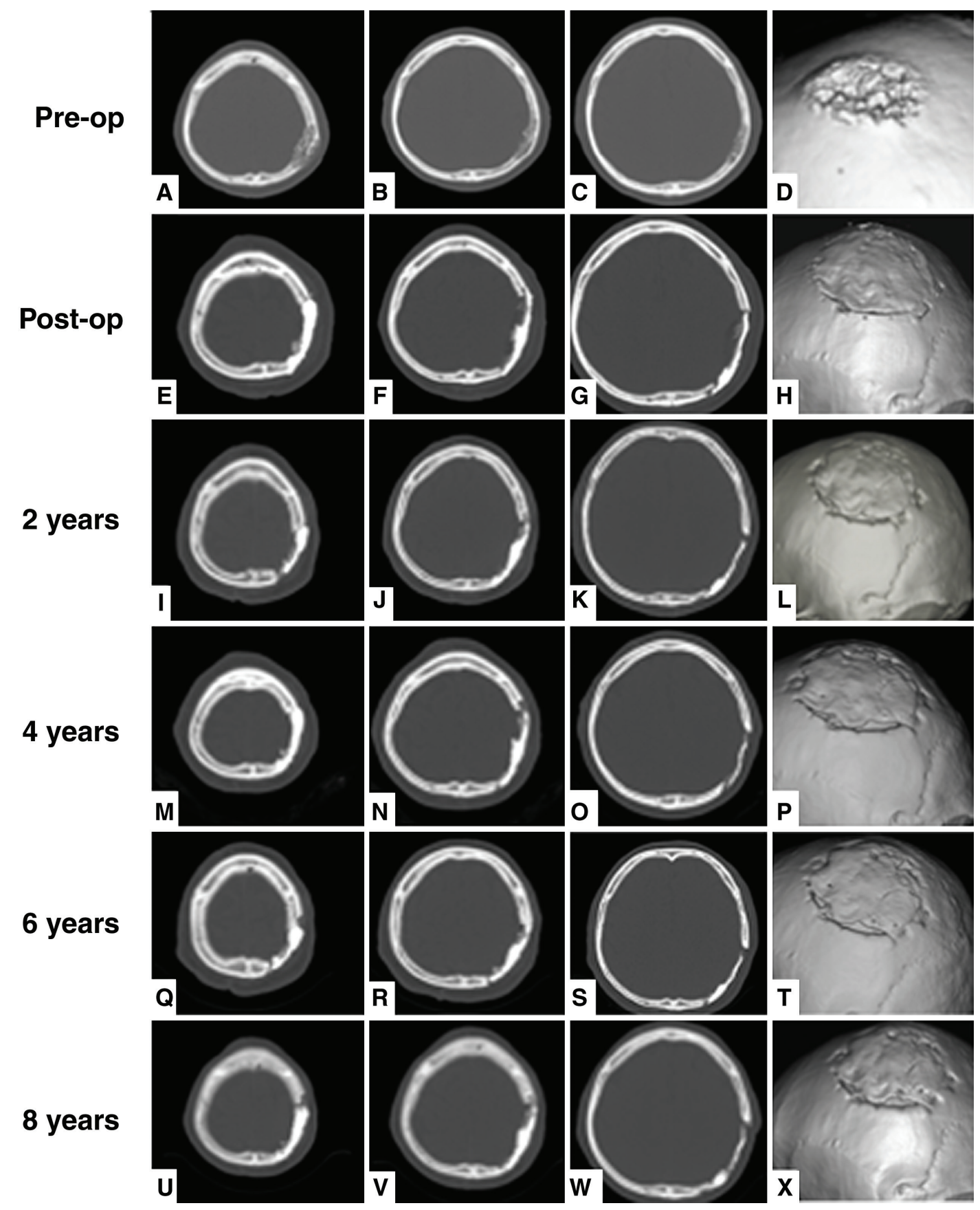

Fig. 1 Presurgical CT images (A-D) show intraosseous skull mass in the left parietal bone. Postsurgical CT images (E-H), 2-year follow-up (I-L), 4-year follow-up (M-P), 6-year follow-up (Q-T), and 8-year follow-up (U-X) are shown, respectively. Both split rib grafts (E, F, I, J, M, N, Q, R, U and V) and CPC grafts (G, K, O, S, and W) are well preserved. CPC: calcium phosphate cement.

observation that CPC was not absorbed if it is mixed with a small amount of blood, and porosity of HA was increased by the mixture of a small quantity of blood into CPC, and this addition stimulated osteogenesis in HA. ${ }^{16)}$ Together with the observation of the experimental prefabrication of vascularized bone of porous PMMA in the periosteum using animal model,17) leaving periosteum tissue at the time of rib graft harvesting may be considered for another surgical option. CPC has a low profile of absorption, ${ }^{18)}$ which adds the more protection to the cranium, like our case. The issue whether the autograft technique is cost-effective still remains unclear, as this surgery was carried out by two 

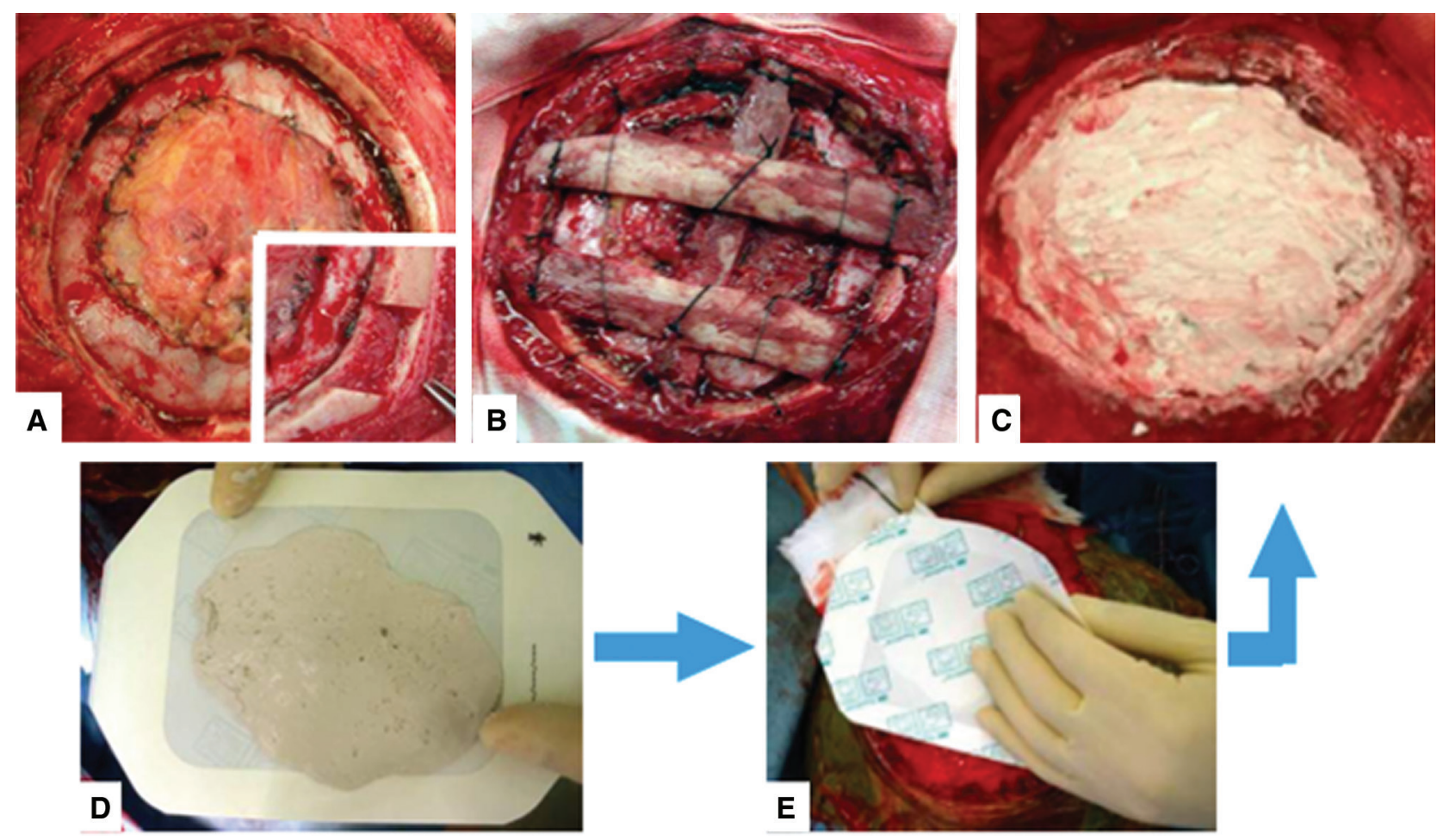

Fig. 2 After $8 \mathrm{~cm} \times 8 \mathrm{~cm}$ of the skull was removed, the dura matter was also incised and free fascia lata graft was applied (A). A tenon is created at the recipient skull ( $\mathrm{A}$, inset). Split rib grafts were arranged into parallel crosses (B). $10 \mathrm{~mL}$ of CPC was prepared into the two medical grade transparent dressings (C). When application of CPC onto the autologous grafts, one side of transparent film was removed (D). Then removed another side of the film, CPC graft was placed on the autologous grafts (E). CPC: calcium phosphate cement.

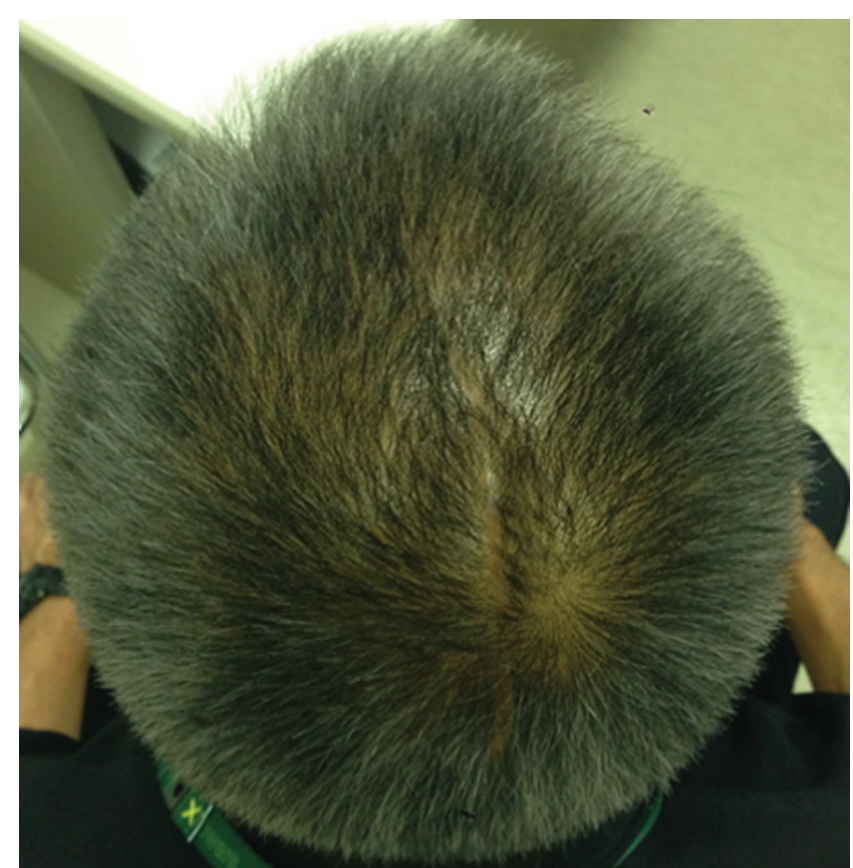

Fig. 3 The head keeps a beautiful shape 8 years after the surgery. surgeons (one neurosurgeon and one plastic surgeon). There may be a criticism that if a cranioplasty is performed by a single surgeon, the cost of the second surgeon (plastic surgeon) may exceed the cost of alloplastic material.

The limitation of our study is the same as the ones in the Catcher's mask cranioplasty or the one in the CPC cranioplasty, that it could not be applied in the site with a higher risk of infection or at irradiated site. We consider that cranial defects for these conditions with donor site morbidity require technically complex reconstruction.

\section{Conclusion}

Our methods, CPC cranioplasty augmented by the Catcher's mask cranioplasty, fulfilled the requirements of aesthetics and in-situ plasticity in a larger cranial defect.

\section{Conflicts of Interest Disclosure}

The authors have no conflicts of interest to declare. 


\section{References}

1) Oliver JD, Banuelos J, Abu-Ghname A, Vyas KS, Sharaf B: Alloplastic cranioplasty reconstruction: a systematic review comparing outcomes with titanium mesh, polymethyl methacrylate, polyether ether ketone, and norian implants in 3591 adult patients. Ann Plast Surg 82: S289-S294, 2019

2) Williams LR, Fan KF, Bentley RP: Custom-made titanium cranioplasty: early and late complications of 151 cranioplasties and review of the literature. Int J Oral Maxillofac Surg 44: 599-608, 2015

3) Moles A, Marie Heudes P, Amelot A, et al.: Longterm follow-up comparative study of hydroxyapatite and autologous cranioplasties: complications, cosmetic results. osseointegration World Neurosurg 111: e395-e402, 2018

4) Kihlström Burenstam Linder L, Birgersson U, Lundgren K, Illies C, Engstrand T: Patient-specific titanium-reinforced calcium phosphate implant for the repair and healing of complex cranial defects. World Neurosurg 122: e399-e407, 2019

5) Afifi AM, Gordon CR, Pryor LS, Sweeney W, Papay FA, Zins JE: Calcium phosphate cements in skull reconstruction: a meta-analysis. Plast Reconstr Surg 126: 1300-1309, 2010

6) Wiltfang J, Kessler P, Buchfelder M, Merten HA, Neukam FW, Rupprecht S: Reconstruction of skull bone defects using the hydroxyapatite cement with calvarial split transplants. J Oral Maxillofac Surg 62: 29-35, 2004

7) Takumi I, Akimoto M, Morita A: Use of an inverted and segmented galea-calvarial flap for the simultaneous reconstruction of the frontal sinus and forehead: a new supplementary method of reconstructive cranial surgery. J Nippon Med Sch 83: 125-129, 2016

8) Takumi I, Akimoto M: Advantage of Catcher's mask cranioplasty for post-surgical infectious skin trouble. Childs Nerv Sys 25: 493-495, 2009

9) Takumi I, Akimoto M: Catcher's mask cranioplasty for extensive cranial defects in children with an open head trauma: a novel application of partial cranioplasty. Childs Nerv Sys 24: 927-932, 2008

10) Eufinger H, Wittkampf AR, Wehmöller M, Zonneveld FW: Single-step fronto-orbital resection and reconstruction with individual resection template and corresponding titanium implant: a new method of computer-aided surgery. J Craniomaxillofac Surg 26: 373-378, 1998

11) Broeckx CE, Maal TJJ, Vreeken RD, Bos RRM, Ter Laan M: Single-step resection of an intraosseous meningioma and cranial reconstruction: technical note. World Neurosurg 108: 225-229, 2017

12) Young CC, Hanak BW, Patel AP, Sekhar LN: Rapid intraoperative in situ synthetic cranioplasty. World Neurosurg 112: 161-165, 2018

13) Durham SR: Correction of large $\left(>25 \mathrm{~cm}^{2}\right)$ cranial defects with "reinforced" hydroxyapatite cement: technique and complications. Neurosurgery 52: 842-845; discussion 845, 2003

14) Morales-Gómez JA, Garcia-Estrada E, Leos-Bortoni JE, et al.: Cranioplasty with a low-cost customized polymethylmethacrylate implant using a desktop 3D printer. J Neurosurg: 1-7, 2018. (ahead of print)

15) Cabbad NC, Stalder MW, Arroyave A, Wolfe EM, Anthony Wolfe S: Autogenous bone cranioplasty: review of a 42-year experience by a single surgeon. Plast Reconstr Surg 143: 1713-1723, 2019

16) Koizumi $T$, Komuro Y: Influence of mixing blood with calcium phosphate bone paste on hardening. J Craniofac Surg 22: 329-332, 2011

17) Cheng MH, Brey EM, Allori AC, et al.: Periosteum-guided prefabrication of vascularized bone of clinical shape and volume. Plast Reconstr Surg 124: 787-795, 2009

18) Shiotani A, Okubo K, Saito K, et al.: Injection laryngoplasty with calcium phosphate cement. Otolaryngol Head Neck Surg 140: 816-821, 2009

Corresponding author: Ichiro Takumi, MD, PhD Department of Neurosurgery, St. Marianna University School of Medicine, 2-16-1 Sugao, Miyamae-ku, Kawasaki, Kanagawa 216-8511, Japan. e-mail: takumi@marianna-u.ac.jp 